\title{
Leonhard'sche „Aufteilung der endogenen Psychosen und ihre differenzierte Ätiologie
}

Karl Leonhard, Hrsg. Helmut Beckmann: Aufteilung der endogenen Psychosen und ihre differenzierte Ätiologie, 8. Auflage, Georg-Thieme-Verlag Stuttgart - New York 2003, 427 Seiten, gebunden. ISBN 3-13-128508-7. 49,95€

$E$ s ist ein großes Verdienst von Beckmann, Würzburg, sich um die Leonhard'sche „Aufteilung der endogenen Psychosen und ihre differenzierte Ätiologie“ jahrzehntelang zu kümmern. Ist doch für viele Psychiater die Klassifikation nach Leonhard ein gewaltiger Fortschritt, aber leider immer noch nicht genügend anerkannt.

Man muss Leonhard umso mehr schätzen, als er selber einräumt, für seine von ihm erhobenen Befunde evtl. auch andere Erklärungsmöglichkeiten $\mathrm{zu}$ finden. Ein Standpunkt von Größe und nebenbei bemerkt, von K. Popper als Kernstück aller Wissenschaftlichkeit wiederholt herausgehoben.

Die Realisierung Leonhard'scher Gedanken und Systeme sieht allerdings nicht rosig aus, wenn man nur an die unsinnigen Diagnosenschemata (ICD 10 usw.) denkt, die ein deutlicher Rückschritt in unserem Fach sind und dringend revidiert werden müssten. Manchmal hat man den Eindruck, dass die Psychiater auch deswegen von Leonhard abrücken, weil es sie zu geistigen Anstrengungen veranlasst, die für manche eben lästig sind.

Eine „Beschreibung“ ist nicht möglich, weil das Buch bekannt ist und auch in der 6 . Auflage alles das zeigt, was Leonhard gesagt und gedacht hat. Dem ist nur hinzu zu fügen, dass sich deutsche Psychiater i.S. Beckmanns mehr als bisher darum kümmern müssten.

F. Reimer, Weinsberg 